\title{
EDITORIAL
}

\section{NECROTIZING ENTEROCOLITIS: PREVENTION IS THE ULTIMATE GOAL}

\author{
Mário Cícero Falcão
}

RHCFAP/3092

For us neonatologists, research about necrotizing enterocolitis always incites great curiosity, because our understanding of its physiopathology is largely speculative, and this illness haunts the neonatal intensive care unit. Nowadays, as viability increases, the prevalence of necrotizing enterocolitis is becoming more important because we are taking care of more immature and lower birth-weight infants.

Many conditions inherent to prematurity may provoke enterocolitis. However, its pathogenesis is complex and multifactorial; therefore, it is important to emphasize the risk factors besides prematurity: perinatal asphyxia with hypoxia-ischemiareperfusion injury, enteral feeding, and bacterial colonization and/or translocation of the gastrointestinal tract. Prematurity is the single greatest risk factor; decreasing gestational age is associated with an increased risk for necrotizing enterocolitis. Preliminary analysis of the other risk factors shows that immaturity may provoke all of them, since prematurity and asphyxia are commonly associated.
Moreover, providing appropriate nutrition for premature infants is not a simple task, and sometimes the absence of human milk obliges us to prescribe infant formulas based on cow milk proteins. These artificial diets could theoretically cause the aggression of immature intestinal mucosa, especially if ischemia is present as a result of asphyxia.

In addition, the precise relationship between enteral feeding and the occurrence of necrotizing enterocolitis has not yet been defined, but it is still accepted that the interaction between substrate delivered into the gastrointestinal tract and luminal bacteria plays an important role in the onset of mucosal injury.

Another factor that must be highlighted is intestinal bacterial colonization. Microorganisms from vaginal delivery and maternal contact colonize the newborn. The feeding type also presents a primordial aspect in this colonization. Premature infants are sometimes delivered by cesarean section, which changes their bacterial colonization. Additionally, contact with the mother (skin to skin) is post- poned because the intra-uterine environment must be mimicked by incubators in the neonatal intensive care unit, where bacterial colonization will be totally different and will include more aggressive microorganisms.

Bacterial colonization can also be altered by artificial respirators and many procedures that are commonly used to keep immature infants alive, which unfortunately increases morbidity and mortality and perhaps initializes necrotizing enterocolitis.

In this context, several studies have been conducted focusing on clarifying the pathogenesis of enterocolitis, emphasizing the initial inflammatory response. Much knowledge has been accumulating about local and systemic inflammatory mediators. Experimental studies have played an important role in the elucidation of several mediators, such as tumor necrosis factor and platelet-activating factor. Until now, all experiments have had a premise of an illness model, i.e., that necrotizing enterocolitis was already in progress, and the results of the experiments would be used in its treatment. 
According to the updated approach in medicine, which is focused on prevention, studies should be performed before the onset of enterocolitis. By analogy with previous studies using antenatal steroids to enhance lung maturation, it can be supposed that antenatal steroids would be efficient in other sites, including intestinal mucosa. Using antenatal steroids in premature infants could enhance gastrointestinal maturation, leading to a reduced incidence of necrotizing enterocolitis, which develops primarily under conditions of gastrointestinal immaturity.

See related article - page 243 